\begin{tabular}{|c|c|}
\hline Title & Towards the first-principles investigation of Ordering Dynamics \\
\hline Author(s) & Mohri, Tetsuo; Ohno, Munekazu; Chen, Y ing \\
\hline Citation & $\begin{array}{l}\text { PRICM 5: Proceedings of the 5th Pacific Rim International Conference on A dvanced Materials and Processing, Pts 1-5, } \\
\text { 3075-3080 }\end{array}$ \\
\hline Issue Date & 2005 \\
\hline Doc URL & http:/hdl.handle.net/2115/16873 \\
\hline Rights & the original is available online at www.scientific.net \\
\hline Tyре & article (author version) \\
\hline Note & Materials Science Forum, V olume 475-479 \\
\hline File Information & PRICM2005.pdf \\
\hline
\end{tabular}

Instructions for use 


\title{
Towards the first-principles investigation of Ordering Dynamics
}

\author{
Tetsuo Mohri ${ }^{1, a}$, Munekazu Ohno ${ }^{1 *, b}$ and Ying Chen ${ }^{2, c}$ \\ 1) Division of Materials Science and Engineering, Graduate School of Engineering, \\ Hokkaido University, Sapporo 060-8628, JAPAN \\ 2) Department of Quantum Engineering and System Science, \\ School of Engineering, The University of Tokyo, Tokyo 113-8655, JAPAN \\ $\left.1^{*}\right)$ present address: Technical University, Clausthal, Robert-Koch Str. 42, \\ D-38678, Clausthal-Zellerfeld, Germany
}

ªtmohri@eng.hokudai.ac.jp, ${ }^{b}$ munekazu-ohno@tu-clausthal-de, 'cying@q.t.u-tokyo.ac.jp

Keywords: Phase Field Method, Cluster Variation Method, First-principles calculation, Cluster Expansion method, Ordering dynamics, Fe-Pd system, L1 $1_{0}$ ordered phase

\begin{abstract}
Phase Field Method (PFM) is hybridized with Cluster Variation Method (CVM) to investigate the ordering dynamics of $\mathrm{L}_{0}$-disorder transition at atomistic and microstructural scales simultaneously. For this, coarse graining operation is attempted on the inhomogeneous free energy functional of CVM. The resultant gradient energy coefficient is found out to be dependent on temperature and order parameters, which is in marked contrast to a conventional PFM formalism. Electronic structure total energy calculations for Fe-Pd system are incorporated to the hybridized scheme and the first principles calculation of microstructural evolution process is attempted.
\end{abstract}

\section{Introduction}

Phase Field Method(PFM)[1] has been recognized as a powerful means to investigate microstructural evolution process of an alloy system. The conventional PFM, however, is a phenomenological scheme in the sense that the choice of ordered parameters and description of the free energy leave ambiguities.

Cluster Variation Method(CVM)[2] has been employed for calculating a phase diagram. The advantage of CVM is that the wide range of atomic correlations can be incorporated in the free energy functional and, therefore, the transition temperature as well as the detailed information of local atomic configuration are obtained with high accuracy.

In the present study, we attempted to hybridize CVM within the local free energy density of the PFM. In view of the fact, however, that the conventional PFM is formulated for continuum media whereas CVM free energy is defined on a discrete lattice, scaling properties should be carefully addressed. Hence, coarse graining operation is performed on the inhomogeneous free energy density of CVM, which enables one to introduce atomistic interaction energies obtained by electronic structure calculations into PFM formalism. The purpose of the present study is to attempt the first principles calculation of ordering dynamics based on the new formalism of hybridized model. A particular focus is placed on time evolution/devolution of Anti Phase Boundary(APB) associated with $\mathrm{L1}_{0}$-disorder transition of Fe-Pd system.

\section{Ground state analysis}

By employing FLAPW[3] within GGA[4], the total energies of a set of ordered compounds are calculated as a function of lattice constant, $r$. [5-7] Those ordered phases are $\mathrm{Fe}_{3} \mathrm{Pd}$ and $\mathrm{FePd}_{3}$ with $\mathrm{L}_{2}$ structure and FePd with $\mathrm{L}_{0}$ structure in addition to $\mathrm{Fe}$ and $\mathrm{Pd}$ with fcc structure. In view of the fact that the ground state of Fe is not fcc but is bcc, the additional calculation is carried out for bcc-Fe. It is found that the magnetism plays an essential role in the ground state phase stability and it is, therefore, necessary to employ spin polarized calculation.

With reference to the segregation limit which is defined as the concentration average of the total 


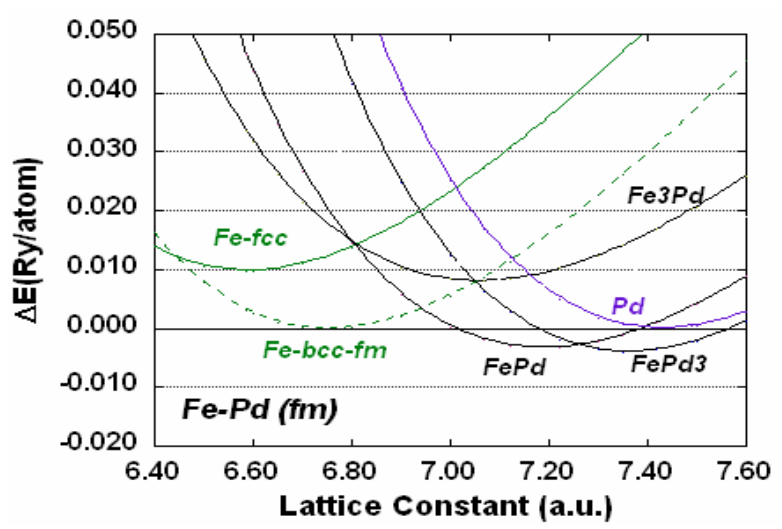

Fig. 1 Heats of formation of six phases for Fe-Pd system [5-7]

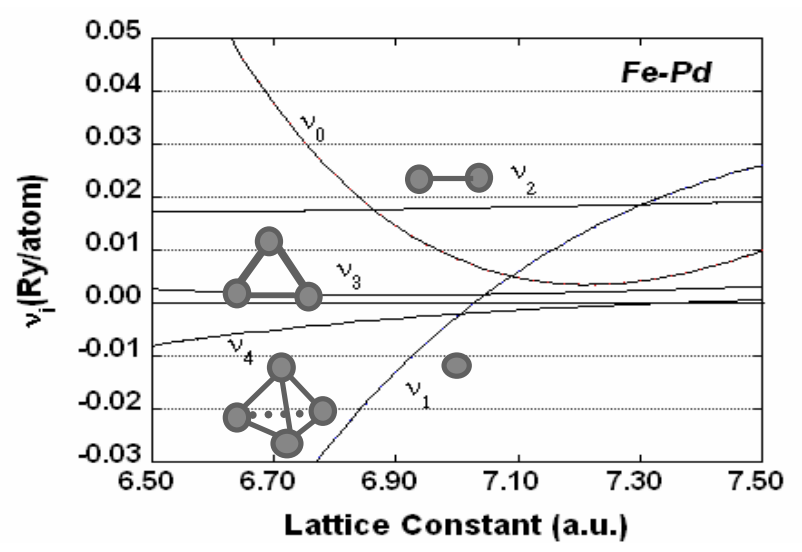

Fig. 2 Effective cluster interaction energies. Subscript $i$ indicates $i$-point cluster. [5-7]

energies of bcc-Fe and $\mathrm{Pd}$, one can obtain the heats of formation $\Delta E^{(m)}(r)$ where $m=1-5$ refer to Fe, $\mathrm{Fe}_{3} \mathrm{Pd}, \mathrm{FePd}, \mathrm{Fe}_{3} \mathrm{Pd}$ and $\mathrm{Pd}$. The results are shown in Fig. 1[5-7]. One can confirm that the heats of formation of $\mathrm{FePd}$ and $\mathrm{FePd}_{3}$ are negative while that of $\mathrm{Fe}_{3} \mathrm{Pd}$ is positive, indicating the correct tendency of the phase stability. Note that the lattice constant of bcc-Fe is smaller than those of other phases. Hence, the lattice constant of fcc-equivalent is calculated for bcc-Fe in order to facilitate the comparison. It is also noted that in view of the closeness of the calculated tetragonal distortion (0.97) to unity for $\mathrm{L} 1_{0}$ ordered phase, the tetragonality is ignored in the following calculations.

By operating the Cluster Expansion[8],

$$
v_{i}(r)=\sum_{m}\left\{\xi_{j}^{m}\right\}^{-1} \cdot \Delta E^{(m)}(r)
$$

the effective cluster interaction energies, $v_{i}$, are derived and plotted in Fig. 2[5-7], where $i=0-4$ correspond to null, point, nearest neighbor(n.n.) pair, n.n. triangle and n.n. tetrahedron clusters. In Eq.1, $\xi_{j}^{m}$ is the correlation function[9] which is defined as the ensemble average of spin operator which takes either +1 or -1 depending upon $\mathrm{Fe}$ or $\mathrm{Pd}$, respectively, and describes the atomic arrangements of cluster $j$ involved in phase $m$. One sees that the pair interaction $\left(v_{2}\right)$ is far larger than three $\operatorname{body}\left(v_{3}\right)$ and four body $\left(v_{4}\right)$ interaction energies, which is a characteristic feature of metallic alloy systems of which cohesion is mainly dominated by central forces.

Once the effective cluster interaction energies are derived, the internal energy $\Delta E(r)$ of a given phase can be written as

$$
\Delta E(r)=\sum_{j} v_{j} \cdot \xi_{j} \quad,
$$

where the set of correlation functions $\left\{\xi_{j}\right\}$ are unknown quantities and are regarded as variational parameters in the calculation of thermodynamic equilibrium state as will be described in the next section.

\section{CVM free energy and Phase Diagram}

In order to investigate the phase equilibria at finite temperatures, entropy term is formulated based on Cluster Variation Method. In the present study, tetrahedron approximation[10] is employed and the entropy formula is given by the following equation.

$$
S=k_{B} \cdot \ln \frac{\prod_{i, j}\left\{N y_{i j} !\right\}^{6} \cdot\{N !\}}{\prod_{i}\left\{N x_{i} !\right\}^{5} \cdot \prod_{i, j, k, l}\left\{N w_{i j k l} !\right\}^{2}},
$$




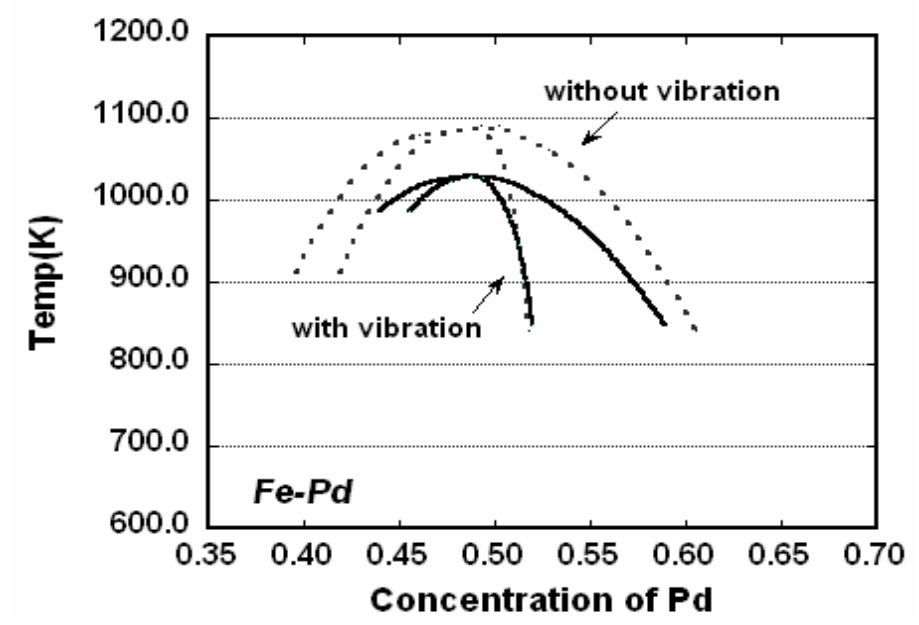

Fig. 3. $\mathrm{L} 1_{0}$-disorder phase diagram for Fe-Pd calculated from the first-principles. The solid(dotted) lines are obtained with (without) lattice vibration effects. The calculated transition temperatures are $1030 \mathrm{~K}$ (with vibration) and $1080 \mathrm{~K}$ (without vibration), respectively.[6,7]

where $x_{i}, y_{i j}$ and $w_{i j k l}$ are cluster probabilities of finding the atomic arrangements specified by subscript(s) on a point, pair and tetrahedron clusters, $N$ the total number of lattice point and $k_{B}$ is the Boltzmann constant.

Together with the internal energy $\Delta E(r)$ in Eq. (2), the free energy is obtained as

$$
g=\Delta E\left(r,\left\{\xi_{i}\right\}\right)-T \cdot S\left(\left\{x_{i}, y_{i j}, w_{i j k l}\right\}\right) .
$$

It is noted that the cluster probabilities, $x_{i}, y_{i j}$ and $w_{i j k l}$, are related to a set of correlation functions $\left\{\xi_{j}\right\}$ through the linear transformation, and, therefore, the free energy $g$ is symbolically written as $g\left(T, r,\left\{\xi_{i}\right\}\right)$. Then, the equilibrium state is determined by minimizing the free energy with respect to a set of correlation functions and a lattice constant under a given temperature $T$,

$$
\left.\frac{\partial g}{\partial\left\{\xi_{j}\right\}}\right|_{T, R, j \neq i}=0 \quad \text { and }\left.\quad \frac{\partial g}{\partial r}\right|_{T,\left\{\xi_{j}\right\}}=0
$$

The extension of entropy and free energy formula for a disordered phase given above to the $\mathrm{L} 1_{0}$ ordered phase is straightforward[10] by distinguishing the sub-lattices. By performing the minimization operation on the free energy of $\mathrm{L} 1_{0}$ ordered phase, which is described in a similar manner to eq.(5), the common tangent construction is carried out to derive the two phase equilibria.

The calculated phase boundaries are demonstrated in Fig. 3[6,7]. The solid (dotted) line indicates the one with (without) lattice vibration effects considered[7] through Debye-Gruneisein model. In the light of the experimental transition temperature of $1023 \mathrm{~K}$, the present results of $1030 \mathrm{~K}$ (with vibration) and $1080 \mathrm{~K}$ (without vibration) provide strikingly good agreements. The congruent composition, however, is located at 50at.\% which is far deviating from the experimental one of 60at.\%. Despite various attempts, this was not corrected and it remains as a future subject to settle this inconvenience.

\section{First-principles Phase Field Method}

Within the PFM, microstructural evolution process is described by the time evolution of a spatial distribution of field variables which constitute the free energy. Field variables are classified into two categories, conserved and non-conserved variables. The concentration, $c$, and Long Range Order parameter (hereafter LRO), are typical examples of each category. 
More precisely, in the PFM, temporal evolution/devolution of conserved and non-conserved field variables are, respectively, described by the following Cahn-Hilliard $(\mathrm{CH})$ equation and the time-dependent-Ginzburg-Landau (TDGL) equation,

$$
\frac{\partial \eta_{i}}{\partial t}=-\nabla\left(-M \nabla \frac{\delta F_{\text {chem }}}{\delta \eta_{i}}\right)
$$

and

$$
\frac{\partial \eta_{i}}{\partial t}=-\sum_{j} L_{i j} \frac{\delta F_{c h e m}}{\delta \eta_{j}}
$$

where $M$ is the mobility and $L_{i j}$ is the relaxation constant. $F_{c h e m}$ is the chemical free energy which is rewritten by the integral form

$$
F_{\text {chem }}=\int\left(f\left[\left\{\eta_{i}\right\}\right]+\sum_{i=1} \kappa_{i}\left(\nabla \eta_{i}\right)^{2}\right) \cdot d V \quad,
$$

where $V$ is a volume of the system, $f$ is the bulk-chemical free energy density for a uniform system and the second term represents interfacial energy with $\kappa_{i}$ as a gradient-energy coefficient. It is noted that the interfacial energy is included in the chemical free energy which is subject to viriational principle to determine the time evolution of field variables. Hence, the interfacial boundary is not a special entity to be separately described but is merely an inhomogeneous localization of field variables.

What is unique to the present approach is to introduce CVM free energy functional, Eq. 4, in the bulk chemical free energy density term in Eq. 8. This operation implicitly induces two spatial scales in the calculation, which is interpreted in the following manner. At each discretized point in the space of PFM in which microstructure is described, ensemble of fcc lattices are defined. The fcc lattices are regarded as homogeneous system and the free energy is described by CVM in terms of correlation functions which constitute a set of field variables. The average values of the correlation functions (field variables) are different from one point to another in the PFM space, and these differences create interfaces in the microstructure. In the following calculations, the main focus is placed on the ordering evolution at a fixed composition of $50 \%$. Hence, $\mathrm{CH}$ equation is not explicitly solved and only TDGL equation is focused,

$$
\frac{\partial \xi_{i}}{\partial t}=-L_{i}\left(\frac{\partial f_{C V M}\left[\left\{\xi_{i}\right\}\right]}{\partial \xi_{i}}-\kappa_{i} \nabla^{2} \xi_{i}\right)
$$

Since the atomistic CVM free energy is incorporated in the microstructural evolution equation of TDGL, this has been termed hybridized model.

Shown in Fig. 4[11]is one of the typical results of the hybridized model. Figs.4(a) and (b) indicate the microstructural evolution with time when the system of a disordered phase is quenched and aged at a temperature at which $\mathrm{L} 1_{0}$ ordered phase is stable. The gray level suggests the square of the long range order parameter, which simulates the Dark Field image of Transmission Micrograph. One sees that the initial disordered phase (Fig. 4a) transforms to $\mathrm{L}_{0}$ ordered phase characterized by the networks of Anti Phase Boundary(APB) (Fig. 4b). Figs. 4(c) and (d) are atomistic arrangements on a $\{001\}$ plane at a fixed point of micrograph marked by a cross in Fig. 4(a) and (b), respectively. These arrangements of atoms are synthesized based on the obtained correlation functions by utilizing Williams's algorithm[12]. One can observe that initial random arrangement(Fig. 4c) corresponding to a disordered phase (Fig. 4a) evolves to an ordered one (Fig. 4d) for $\mathrm{L}_{0}$ phase (Fig. 4b) with some anti-site atoms. The simultaneous calculations of microstructure and atomistic arrangement are unique capability of the present hybridized model. 


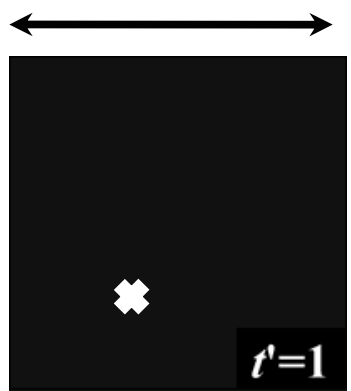

(a)

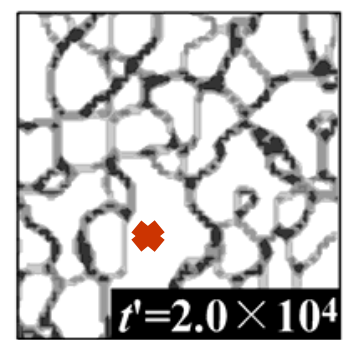

(b)

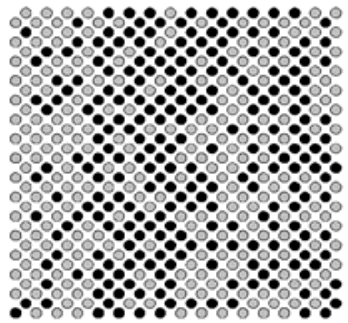

(c)

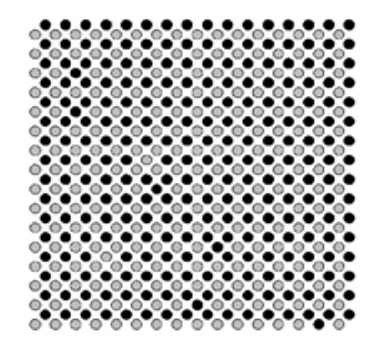

(d)

Fig. 4. Microstructural evolution ((a) and (b)) and atomistic evolution ((c) and (d)) on $\{001\}$. (a) and (c) correspond to disordered phase and (b) and (d) are for $\mathrm{L}_{0}$ ordered phase. An arrow above Fig. 4(a) indicate $x^{\prime}=100$ in the normalized scale.[11]

It has been further demonstrated that the entire evolution kinetics is successfully described as the sum of several kinetic processes including atomistic arrangements, wetting of APB, and the growth and coalescence of APD(Anti Phase Domain), for which characteristic time constants are reasonably separated. Hence the present hybridized scheme proves to describe the multiscale phenomena successfully. The interested readers should read the authors' original article [13].

Despite these successes, spatial scale in the micrograph is normalized as $x^{\prime}=x \cdot \sqrt{\left(N \cdot v_{2}\right) /\left(2 \kappa_{1}^{\alpha}\right)}$, and absolute length scale is not uniquely assigned. Moreover, the crystal orientation is not specified in the micrograph. These inconveniences originate from the insufficient consideration of spatial scaling properties which are closely related to the extension of atomistic interaction energies. In order to describe the two different spatial scales of PFM and CVM, the coarse graining operation is attempted in the following manner.

We start with describing the free energy of an inhomogeneous system by CVM and expand the free energy around the homogeneous system after the coarse graining operation,

$$
\begin{gathered}
F=\sum_{n, m} f_{C V M}\left(\left\{\phi_{s}\left(R_{n}+r_{m}\right)\right\}\right) \\
\cong \sum_{n, m} f_{C V M}\left(\left\{\phi_{s}\left(R_{n}\right)\right\},\left\{r_{m} \cdot \nabla \phi_{s}\left(R_{n}\right)\right\},\left\{r_{m}{ }^{2} \cdot \nabla^{2} \phi_{s}\left(R_{n}\right)\right\}\right) \\
\cong \sum_{n}\left\{N \cdot f_{C V M}\left(\left\{\phi_{s}\left(R_{n}\right)\right\}\right)+\left.\sum_{s, m} \frac{\partial f_{C V M}}{\partial\left(\nabla \phi_{s}\right)}\right|_{0}\left(\nabla \phi_{s}\right)+\left.\sum_{m, s} \frac{\partial f_{C V M}}{\partial\left(\nabla^{2} \phi_{s}\right)}\right|_{0}\left(\nabla^{2} \phi_{s}\right)+\frac{1}{2} \sum_{m, s, s^{\prime}} \frac{\partial^{2} f_{C V M}}{\partial\left(\nabla \phi_{s}\right) \partial\left(\nabla \phi_{s^{\prime}}\right)} \mid\left(\nabla \phi_{s}\right)\left(\nabla \phi_{s^{\prime}}\right)\right\}
\end{gathered}
$$

where $\phi_{s}$ represents a correlation function which is defined for each lattice point specified by two coordinate systems, $\left\{R_{n}\right\}$ and $\left\{r_{n}\right\}$. The former and latter, respectively, identify the coordinate of a coarse grain and the location of an atom within each grain. The description of the inhomogeneous CVM free energy implicitly introduces the interfacial energy in the atomistic level. It is noted that the basic ingredient of the above formalism is traced back to Kikuchi-Cahn's work[14] but the present scheme provides a more generalized framework.

Then, by transforming the sum into the integral form, the chemical free energy of PFM is re-derived as follows,

$$
F=\frac{1}{v_{a}} \int\left[f_{C V M}\left[\left\{\xi_{i}\right\}\right]+\sum_{s, s^{\prime}, i} \kappa_{s, s^{\prime}, i}\left(\nabla_{i} \xi_{s}\right)\left(\nabla_{i} \xi_{s^{\prime}}\right)\right] \cdot d V
$$

which automatically provides the gradient energy coefficient which depends on the cluster 

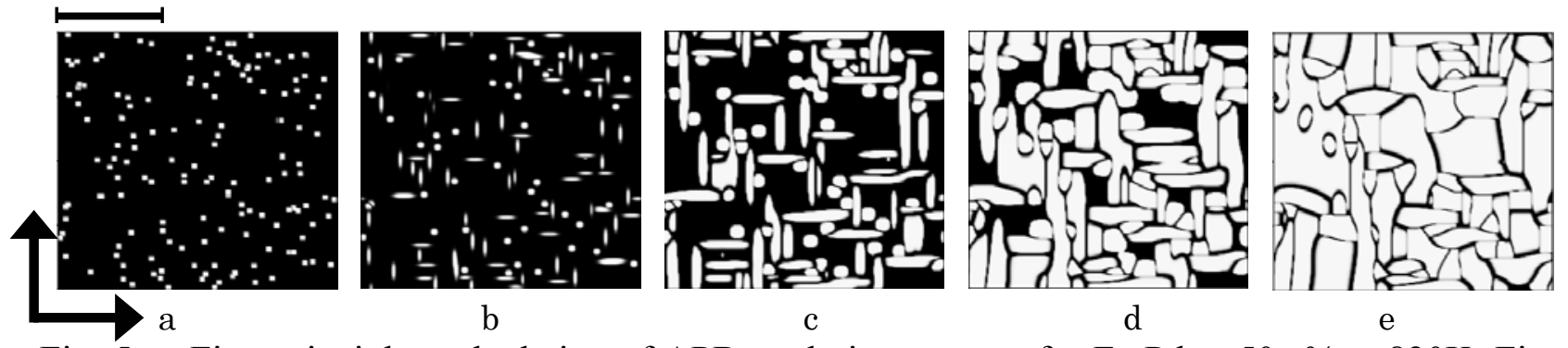

Fig. 5 First-principles calculation of APB evolution process for Fe-Pd at 50at $\%$ at $820 \mathrm{~K}$. Figs.5 a-e correspond to time $t^{\prime}=0.01,0.83,3.59,7.04$ and 16.70 , respectively. The bar above Fig.5a indicates the length of $100 \mathrm{~nm}$ and the arrows suggest [100] and [010] directions.

probabilities (short range order parameters) and temperature,

$$
\kappa_{s, s^{\prime}, i}=\frac{1}{2} N k_{B} T \sum_{t, J}\left(\frac{1}{2^{q(t)}}\right)^{2} \frac{q^{\prime}(t)}{x_{J}^{t}}\left[V_{s, t, i}^{b} \cdot V_{s^{\prime}, t, i}^{b}-\frac{1}{2}\left(V_{s, t, i}^{c} \cdot V_{s^{\prime}, t}^{a}+V_{s^{\prime}, t, i}^{c} \cdot V_{s, t}^{a}\right)\right]
$$

where $V$ is termed $\mathrm{V}$-matrix and is given as the sum of products of correlation functions.

The microstructural evolution for Fe-Pd system at 50\% which is quenched from $1020 \mathrm{~K}$ in the disordered state down to $820 \mathrm{~K}$ in the $\mathrm{L}_{0}$ ordered region (see Fig.3) is calculated. The results are shown in Fig. 5. Since it was confirmed that $\mathrm{T}=820 \mathrm{~K}$ is in the nucleation-growth regime, we first assign nuclei by generating random numbers and the assumption of site-saturation is imposed. One sees that with time the characteristic microstructure of APB is formed. Most importantly, it is noted that the spatial scale and crystallographic orientation are uniquely fixed.

Finally it is pointed out that two major subjects must be settled in the future. One is the effects of the 2 nd nearest neighbor pair interactions. As was reported[6,7], the magnitude of the $2 \mathrm{nd}$ n.n. pair interaction is about $10 \%$ of the nearest neighbor pair interaction and this effect is not negligible. Rather strong anisotropy in the resultant microstructure may be originated from the neglect of the 2nd n.n. pair interaction. Secondly, the absolute value is not assigned to time, and the time scale is normalized as $t^{\prime}=N v_{2} L_{1}^{\alpha} t$. For this, we need to derive the relaxation constants from the atomistic level, which is one of the most challenging subjects.

\section{References}

[1] L.-Q. Chen: A. Rev. Mat. Res. 32 (2002), 113.

[2] R. Kikuchi: Phys. Rev., 81 (1951), 998.

[3] H.J.F.Jansen and A.J. Freeman: Phys. Rev. B30(1984), 561.

[4] J.P. Perdew,J.A. Chevary, S.H. Vosko, K. A. Jackson, M.R. Pederson, D.J. Singh and C. Fiolhais: Phys. Rev. B46 (1992), 6671.

[5] Y. Chen, T. Atago and T. Mohri: J.Phys. Condens. Matter. 14(2002), 1903.

[6] T. Mohri and Y. Chen: J. Alloys and Compounds (2004), in print.

[7] T. Mohri and Y. Chen: Mat. Trans.(2004) in print.

[8] J.W. Connolly and A.R. Williams: Phys. Rev. B27(1983), 5169.

[9] T. Mohri, J.M. Sanchez and D. de Fontaine: Acta Metall.33(1985), 1171.

[10] R. Kikuchi: 1974 J. Chem. Phys.,60 (1974), 1071.

[11] M. Ohno and T. Mohri: Mat. Trans.42(2001), 2033.

[12] R.O. Williams: Report No.ORNL-5140, Oak Ridge National Lab., Oak Ridge, TN (1976).

[13] M. Ohno and T. Mohri: Phil. Mag. 83(2003), 315.

[14] R. Kikuchi and J.W. Cahn: J. Phys. Chem. Solids. 23(1962), 137. 